\title{
FINITENESS CONDITIONS FOR MATRIX SEMIGROUPS
}

\author{
YECHEZKEL ZALCSTEIN
}

\begin{abstract}
Classical theorems of Schur and Burnside on finiteness of matrix groups are generalized to regular and inverse matrix semigroups.
\end{abstract}

A group $G$ is periodic iff every element of $G$ has finite order. $G$ is periodic of bounded period iff there is a bound on the orders of all elements of $G$. The following classical theorems give conditions for finiteness of periodic groups of matrices:

BURNSIDE'S THEOREM [3, (36.1)]. A periodic group of complex $n \times n$ matrices of bounded periodic is finite.

SCHUR's Theorem [3, (36.2)]. A finitely generated periodic group of complex $n \times n$ matrices is finite.

In [4], we have observed that both theorems generalize trivially to irreducible matrix semigroups and that Burnside's theorem is false in general for semigroups. In this note we prove that Schur's theorem generalizes to regular semigroups and, as a corollary, that Burnside's theorem generalizes to inverse semigroups. Recall that for elements $x, y$ of a semigroup, $y$ is a generalized inverse of $x$ iff $x y x=x$ and $y x y=y$. A semigroup $S$ is a regular (inverse) iff every element of $S$ has a (unique) generalized inverse.

We will need the following theorem.

TheOREM (COUdRAIN-SchüTZENBERGER [2]). Let $S$ be a finitely generated monoid. Assume

(1) $S$ satisfies the descending chain condition on principal two-sided ideals.

(2) For all $x, y$ in $S,\{x, y\} \subset S x \cap y S$ implies $\{x, y\} \subset x S \cap S y$.

(3) All subgroups of $S$ are finite.

Then $S$ is finite.

Received by the editors May 23, 1972.

AMS (MOS) subject classifications (1970). Primary 20M30.

Key words and phrases. Matrix semigroup, Schur theorem, d.c.c. on principal ideals.

(c) American Mathematical Society 1973 
A semigroup $S$ is periodic iff for all $x$ in $S$, there are positive integers $m, r$ such that $x^{m+r}=x^{r} . m$ is called the period of $x . S$ is periodic of bounded period iff there is a bound on the periods of all elements of $S$.

THEOREM. Let $S$ be a finitely generated regular periodic semigroup of $n \times n$ complex matrices; then $S$ is finite.

Proof. We need the following lemma:

LEMMA. A regular semigroup $S$ of $n \times n$ matrices over a field satisfies the descending chain condition on right, left and two-sided principal ideals.

Proof. It will suffice to prove the d.c.c. on principal right ideals since the d.c.c. on principal left ideals follows by a dual argument, and by a theorem of Green [1, vol. 2 (6.49)] the d.c.c. on one-sided ideals implies the d.c.c. on two-sided ideals. It will clearly suffice to prove d.c.c. on principal right ideals generated by elements of the same rank. Since $S$ is regular, every principal right ideal has an idempotent generator. Thus it will suffice to prove that if $E, F$ are distinct idempotent matrices in $S$ of rank $k$, and if $F=E A$, for some $A$ in $S$ (we write arguments on the left), then there in $B$ in $S$ such that $F B=E$. Since null space $(E)$ is a subset of null space $(F)$ and since $E$ and $F$ have the same rank, it follows that $E$ and $F$ have the same null space. Let $M$ be the multiplicative semigroup of all $n \times n$ matrices over the ground field. Then $E$ and $F$ generate the same principal right ideal of $M$ (see [1, vol. 1, p. 57]). Thus there is $C$ in $M$ such that $E=F C$. But then if $B=E, F B=F^{2} C=F C=E$ and so $E$ and $F$ generate the same principal right ideal of $S$. Q.E.D.

Without loss of generality, let $S$ be a monoid. By the Lemma, $S$ satisfies condition (1) of the Coudrain-Schützenberger theorem. Condition (2) is easily seen to hold for all periodic semigroups:

Assume $y=s x, x=y t$ for some $s, t$ in $S$ ( $S$ has an identity). Then $s x t=x$ and, for all positive integers $k, s^{k} x t^{k}=x$. Since $S$ is periodic, for some positive integer $n, e=s^{n}$ and $f=t^{n}$ are idempotents. Thus $x=e x f=$ $e^{2} x f=e x=s^{n-1}(s x)=s^{n-1} y$ and similarly $y=x t^{n-1}$ and so $\{x, y\} \subseteq x S \cap S y$.

Finally, a slight extension of the proof of Schur's theorem yields that $S$ is of bounded period [4, (1.4)]. Let $G$ be a subgroup of $S$. Then $G$ is of bounded period and thus finite by Burnside's theorem. It thus follows by the Coudrain-Schützenberger theorem that $S$ must be finite.

Corollary 1. A periodic inverse semigroup $S$ of $n \times n$ complex matrices is completely reducible.

Proof. The proof follows along the lines of [3, (36.3)]. Let $x_{1}, \cdots, x_{r}$ be a maximal set of linearly independent elements of $S$. Let $T$ be the inverse subsemigroup of $S$ generated by $x_{1}, \cdots, x_{r}$ and their generalized inverses. 
$T$ is finite by the theorem and thus, by a theorem of Munn [1, (5.25)], $T$ is completely reducible. It follows easily that $S$ is also completely reducible.

Corollary 2 (Generalized Burnside TheOREM). A periodic inverse semigroup $S$ of $n \times n$ complex matrices of bounded period is finite.

Proof. By Corollary $1, S$ is completely reducible; thus it will suffice to prove the assertion for irreducible semigroups. But this is proved in $[4,(1.2)]$.

\section{REFERENCES}

1. A. H. Clifford and G. B. Preston, The algebraic theory of semigroups. Vols. 1, 2, Math. Surveys, no. 7, Amer. Math. Soc., Providence, R.I., 1961, 1967. MR 24 \#A2627; MR 36 \#1558.

2. M. Coudrain et M. P. Schützenberger, Une condition de finitude des monoides finiment engendrés, C.R. Acad. Sci. Paris Sér. A-B 262 (1966), A1149-A1151. MR 33 \#2742.

3. C. W. Curtis and I. Reiner, Representation theory of finite groups and associative algebras, Pure and Appl. Math., vol. 11, Interscience, New York, 1962. MR 26 \#2519.

4. Y. Zalcstein, Studies in the representation theory of finite semigroups, Trans. Amer. Math. Soc. 161 (1971), 71-87.

Datalogy Institute, University of Copenhagen, Universitetsparken 5, DK-2100 Copenhagen, Denmark

Current address: Department of Computer Science, State University of New York, Stony Brook, New York 11790 